\title{
Hydrogen detection in organic gas mixtures based on analyzing the transient response.
}

\begin{abstract}
Purpose - The purpose of this paper is to explore the ability of capillary-attached gas sensor (CGS) in detecting components of gas mixtures, including a volatile organic gas and hydrogen in a wide range of concentrations. Design/methodology/approach - Diverse feature extraction and classification techniques were employed to analyze the response of CGS when applied to different mixtures. Findings - It was observed that the response of CGS to the above gas mixtures could be distinguishable. While evaluating the results of the classification technique, it was implied that hydrogen, in the presence of the volatile organic gases, could be detected perfectly by analyzing the response of the CGS. Separating techniques, which yielded a high rate of classification, were used to separate mixtures containing hydrogen and organic gases from other organic gas mixtures without hydrogen. Originality/value - The results presented in this paper prove the ability of CGS in fabricating an olfactory machine for analyzing the components of gas mixtures.
\end{abstract}

Keyword: Gases; Sensors; Conduction; Transient response; Diffusion 